\title{
Characterizing Synchronous Writes in Stable Memory Devices
}

\author{
William B. Mingardi ${ }^{1}$, Gustavo M. D. Vieira ${ }^{1}$ \\ ${ }^{1}$ DComp - CCGT - UFSCar \\ Sorocaba, São Paulo, Brasil \\ williammingardi@gmail.com, gdvieira@ufscar.br
}

\begin{abstract}
Distributed algorithms that operate in the fail-recovery model rely on the state stored in stable memory to guarantee the irreversibility of operations even in the presence of failures. The performance of these algorithms lean heavily on the performance of stable memory. Current storage technologies have a defined performance profile: data is accessed in blocks of hundreds or thousands of bytes, random access to these blocks is expensive and sequential access is somewhat better. File system implementations hide some of the performance limitations of the underlying storage devices using buffers and caches. However, fail-recovery distributed algorithms bypass some of these techniques and perform synchronous writes to be able to tolerate a failure during the write itself. Assuming the distributed system designer is able to buffer the algorithm's writes, we ask how buffer size and latency complement each other. In this paper we start to answer this question by characterizing the performance (throughput and latency) of typical stable memory devices using a representative set of current file systems.
\end{abstract}

\section{Introduction}

Paxos [Lamport 1998, 2006] is a distributed consensus algorithm for asynchronous distributed systems. It can be used to create replicas of distributed services to ensure greater availability of the service as a whole [Schneider 1990]. This algorithm and other similar consensus algorithms are used in mission critical systems around the world [Chandra et al. 2007, Hunt et al. 2010].

The Paxos algorithm was designed to tolerate faults assuming a fail-recovery fault model, in which the processes that compose the distributed system can fail by crashing and later recover and return to operation [Cachin et al. 2011]. When one of these failures occurs the process stops operating and loses all state stored in main memory. It can later return to normal operation, but can only rely on state stored in stable memory, such as the hard disk. This failure model is very interesting because it is a suitable representation of the way computers crash, are restarted and reload the operating system and running applications.

Paxos and similar consensus algorithms that operate in the fail-recovery model rely on the state stored in stable memory to guarantee the irreversibility of operations even in the presence of failures. This irreversibility is crucial for the consistency of these distributed algorithms [Lamport 2006]. However, accesses to stable memory are usually much slower than accesses to main memory, and algorithms in the fail-recovery model minimize the use of this costly resource. For example, Paxos has two writes to stable 
memory in its critical path [Lamport 1998]. This way, performance of distributed algorithms in the fail-recovery model will lean heavily on the performance of stable memory.

Currently there are two main implementations of stable memory in use: spinning disks and flash memory. Both technologies are radically different, but have a similar performance profile: data is accessed in blocks of hundreds or thousands of bytes, random access to these blocks is expensive and sequential access is somewhat more efficient [Ruemmler and Wilkes 1994, Min et al. 2012, Chen et al. 2009]. Access to these devices is mediated by the operating system using the file system abstraction. File system implementations take the performance profile of storage devices into account, and are able to hide some of the performance limitations of the underlying device using buffers and caches. However, fail-recovery distributed algorithms require that the data being written is committed to disk before proceeding to be able to tolerate a failure during the write itself. As a consequence, every write must be a synchronous write, usually achieved by a call to fsync or similar system call. Issuing a synchronous write, however, conflicts with most strategies employed by file systems to hide the performance cost of stable memory [Jannen et al. 2015, Yeon et al. 2018].

Considering the limitations of file systems, we ask how can the designer of a distributed system maximize the performance of the available storage devices. To maximize something it is necessary to first establish appropriate metrics. If we only consider throughput of the storage device, its physical implementation and the way file systems are implemented suggest that we should write as much data as possible in any single synchronous write. This way, the file system will be able to fill its buffers, to allocate relatively large extents of contiguous blocks and the device controller will have enough data to optimize the execution of the write. However, from the point of view of the distributed algorithm, a big write will mean a larger latency for the operation to complete. Making the question much more interesting is the fact that due to the way file systems are implemented, this latency increase is not directly proportional to the amount of data being written. Specifically, synchronous writes of only a few bytes each will reduce the throughput of the disk to almost zero, while large writes will hardly affect the latency of the operation.

Thus, if the distributed system designer is able to buffer the algorithm's writes to stable memory [Vieira and Buzato 2010] and wants to use storage devices at optimum capacity, we need to understand exactly how buffer size and latency complement each other. To this end, we should evaluate the full programming environment, including operating system, file system and storage device. In this paper we start this investigation by characterizing the performance (throughput and latency) of typical stable memory devices, a spinning disk and a solid state drive, using a representative set of current file systems. Many works evaluate file system and storage device performance [Chen et al. 2009, Min et al. 2012, Jannen et al. 2015, Sweeney et al. 1996, Mathur et al. 2007, Rodeh et al. 2013, Lee et al. 2015], however these works usually assume the file system will be able to handle any type of operation the application requires. This work is different in the sense that we investigate how the distributed system designer can help the file system and storage device better handle the use pattern created by distributed algorithms.

This paper is organized as follows. In Section 2 we describe the basic assumptions of our research and related work. Section 3 describes our experimental setup and 
Section 4 shows the experimental data and analysis. We present some concluding remarks in Section 5.

\section{Background}

\subsection{Synchronous Writes}

Storage devices are orders of magnitude slower than main memory. Random access of data at the byte level is prohibitively expensive and the best performance of these devices can be achieved by accessing data in sequential blocks of hundreds or thousands of bytes [Ruemmler and Wilkes 1994, Min et al. 2012, Chen et al. 2009]. This reality has shaped the design of file systems for decades. Among the techniques used are caching of recently accessed blocks in memory for reading and writing, and the pre-fetching of blocks that are likely to be accessed in the future [Rosenblum and Ousterhout 1992]. Write caches are particularly useful because they allow successive small writes to happen to the same block while requiring only one single write to the storage device.

The negative consequence of the use of write caches is that the application can't be sure if the data it has written is effectively recorded in the storage device. If a failure occurs after the write is issued but before the data is written to stable memory this write may be lost. For many applications this behavior is acceptable, as a crash means the application has already unexpectedly stopped and it is inconsequential if this failure happened before or after the write. After a potential recovery, that application just redoes the interrupted write. However, some applications that interact with external systems have the requirement that a write they had made must not be forgotten. One simple example of these applications is an automated teller machine that can't undo the fact it has already dispensed money. Distributed algorithms have similar requirements, but instead of money they can't take back messages that were already sent [Lamport 2006].

Operating systems support the implementation of applications that require that a write be committed to stable storage through a fsync or similar system call. This call will force the file system to write to disk all buffers it keeps in memory. Very frequently metadata and caches are also flushed and immediately written to the underlying device [Jannen et al. 2015, Yeon et al. 2018]. This satisfies the requirement of the application, but have a considerable impact on the performance of the file system. Moreover, the application itself can be victim of its own access patterns. To explain how this happens, let's consider the situation of a distributed algorithm that needs to write $x$ bytes for each message it sends, where $x$ is small number. If this application writes synchronously these few bytes, it will have to wait for the write of a complete file system block plus any metadata changes required by the file system. If we assume the latency of writing a block is $l_{b}$ and the latency of metadata update is $l_{m}$, the throughput of this write is:

$$
\frac{x}{l_{b}+l_{m}}
$$

However, if the application is able and decides to batch a group of messages sending them at the same time, it can make a single write corresponding to the record of all messages sent at once. If this group has $y$ messages and the size of bytes written $x y$ is smaller than the file system block size, we can assume the latency $l_{b}+l_{m}$ will remain 
constant. Under this assumption, the throughput of this batched write will be:

$$
\frac{x y}{l_{b}+l_{m}}
$$

that is $y$ times larger than the throughput of recording the send of a single message. Depending on the size of the file system block and the size of this application buffer of $x y$ bytes, the increase of throughput obtained can be orders of magnitude larger than the case where $y=1$. Also, the latency $l_{b}+l_{m}$ can be considered the minimum latency required to perform a synchronous write. We can improve the throughput, but the best we can achieve under ideal conditions is to maintain this minimum latency.

The assumption that $l_{b}+l_{m}$ will remain constant is a bit optimistic, though. At the least, as $x y$ increases eventually the size of the application buffer will exceed the file system block. Moreover, the amount of metadata updates required will increase as the number of file system blocks touched by the write also increases. Then, as we increase $y$ the latency will eventually creep up, matching some of the throughput gains we obtained. The question an application programmer has to face now is how many messages should one batch in an application buffer to increase throughput to an ideal level while not increasing latency disproportionately.

This trade-off of throughput and latency is not new, actually it is at the heart of many computing devices we rely on [Patterson 2004]. What is interesting in this case is that, at least for implementations of distributed algorithms and the current designs for file systems, this trade-off falls squarely in the hands of the application programmer. Two techniques that have been employed at the application level is to use a log-like structure to keep writes sequential and accumulating data in a large enough buffer [Vieira and Buzato 2010]. These are common sense approaches [Patterson 2004], but that lack a solid framework to evaluate their effectiveness.

\subsection{Related Work}

Management of synchronous writes is a problem usually faced by file system designers with respect to metadata consistency [Rosenblum and Ousterhout 1992]. Coalescing write operations in the buffer cache is usually done internally by the file system, instead of the application. For this reason, studies considering the performance of synchronous writes under different application buffer sizes are practically nonexistent.

From the point of view of file system design and implementation, many studies focus on making metadata updates more efficient. One approach is a log-structured file system [Rosenblum and Ousterhout 1992] such as F2FS [Lee et al. 2015]. This file system tries to transform random write patterns in sequential ones by avoiding to change metadata in place. Instead, changes are written in a sequential log of changes, properly indexed for later access. F2FS designers show that synchronous writes make or break a file system performance and that coalescing writes can be very efficient [Lee et al. 2015]. F2FS writes data in chunks of $512 \mathrm{kB}$ while a recent file system such as ext4 [Mathur et al. 2007] usually writes $4 \mathrm{kB}$. F2FS is a very efficient file system, however there is a limit to what the file system can do alone. Many small synchronous writes will kill the performance of any file system, including F2FS, as we show in Section 4.

Research proposing new file systems usually is accompanied by performance evaluations [Sweeney et al. 1996, Mathur et al. 2007, Rodeh et al. 2013, Lee et al. 2015], 
but the focus are workloads that do not include synchronous writes. One exception is [Lee et al. 2015] that shows performance data of a workload with fsync on top of F2FS. However, this evaluation assumes many concurrent threads providing enough data to batch the writes in larger buffers. We are interested in the performance of singlethreaded synchronous writes that reflect more accurately the latency expected by a distributed algorithm. Other examples of works that perform extensive performance evaluations of storage systems, but do not consider synchronous writes, are [Chen et al. 2009] and [Jannen et al. 2015].

Write-optimized file systems such as BetrFS [Jannen et al. 2015] could be used to offer efficient writes to the application, regardless of buffer size. However, these file systems still suffer from many limitations [Jannen et al. 2015] and aren't yet in widespread use. The fsync optimizations present in [Yeon et al. 2018] would help reduce the inherent latency of performing a fsync. This work complements our results in the sense that improvements in fsync latency will be a constant factor reduction of $l_{b}+l_{m}$, but that alone won't change the problem of low throughput with small buffers.

\section{Experimental Characterization}

\subsection{Overview}

We want to characterize the performance of secondary memory, specifically we are interested in the performance of sequential, synchronous writes that ignore the file system buffers. The target application is a distributed algorithm, such as Paxos, that has to perform synchronous writes (fsync) to stable memory before it can proceed with its execution. We also assume that this algorithm processes many requests in parallel and is thus able to assist the file system by coalescing its own writes in larger batches at the cost of increased latency for its operations.

The focus of the characterization is the trade off between stable memory throughput and latency as the application sets the size of its write buffer: the larger the buffer, the larger both throughput and latency. The objective is to give the application designer a tool that can be used to discover, for a combination of stable device implementation and file system, which is the optimal buffer size considering the latency requirements of the application.

We have performed tests with application buffers ranging from $4 \mathrm{kB}$ to $16 \mathrm{MB}$, as these sizes cover the point where latencies start to grow proportionally to the buffer size. The tests write sequentially a new file of $16 \mathrm{MB}$ and we have observed that bigger files do not change the throughput and latency data observed. The experiments were run on different combinations of stable memory technology (spinning disk and flash memory) and files systems (ext4, XFS, BTRFS, F2FS).

\subsection{Iozone}

The tests were run using the Iozone multi-platform file system benchmark tool. ${ }^{1}$ This tool was selected because it is portable to many operating systems, it can run many different tests including reading, writing, rereading and rewriting, besides being highly customizable. We configured the tool to perform a sequential synchronous write test with the following parameters:

\footnotetext{
${ }^{1}$ http://www.iozone.org/
} 
Test type: The test is a sequential write of a file (-i 0$)$.

Buffer size: The buffer sizes tested range from $4 \mathrm{kB}$ to $16 \mathrm{MB}(-\mathrm{a})$.

File size: The file written has $16 \mathrm{MB}$ (-n 16m -g 16m).

Synchronous write: The writes bypass the file system buffers and go directly to disk and the wait time of the synchronous write is added to the operation latency (-o - I -e).

Latency tests: The default for Iozone is to measure throughput, but we also tested the latency of operations (-N).

Unfortunately, the Iozone documentation is not clear about how many iterations it performs when calculating the throughput and latency for each block size. More importantly, we did not have access to the raw data or even indirect measures beyond the average, such as standard deviation. To achieve more rigorous results we decided to run Iozone repeatedly (30 times) and treat the output of each run as a separate data point. We then calculated the average and standard deviation from this data. Our approach was validated when we observed that for some experimental parameters the variation of the readings for many runs of Iozone was considerable (Section 4).

To automate the process of repeatedly running Iozone we created a Bash script that runs the benchmark, collects the data and aggregates the result, calculating the average and standard deviation. Other aspect automated by the script is that Iozone has to be run twice for each data point: the first for throughput and the second for latency. For each run the raw output of Iozone is parsed by Awk and a simple text file describing this run is created, containing for each block size the relevant metric (throughput or latency). After all runs are performed, these files are coalesced in a single file containing the average and standard deviation for each block size of the measurement. Finally, all intermediary data and the final results are archived in a single compacted file to allow future reprocessing of the data, if necessary.

This way, the script created integrates all steps to reproduce our data, and more importantly, is the seed for an automated tool that could be used to inform an application programmer about the behavior of the file system and storage device being used.

\subsection{Experimental Setup}

The tests were run in a desktop computer running 64 bits Linux (Fedora 25), using version 4.12.9 of the Linux kernel. All tested file systems were at the standard versions found in this kernel release. The computer had an Intel Core 26300 processor, with a $1.86 \mathrm{GHz}$ clock, and $4 \mathrm{GiB}$ of RAM. For storage devices to be tested we installed in the computer two units that throughout the text are going to be referenced as HDD and SSD:

HDD: HITACHI HDS72101 7200 rpm hard disk (1 TB),

SSD: Samsung 850 solid state drive (256 GB).

Both drives were connected to a SATA II interface and the tests were run in a dedicated $19 \mathrm{GiB}$ partition in each drive. The drives were both dedicated to the test, there was no system files or user data in them and no competing accesses.

To validate that our experimental setup and data were robust and to assess the portability of the test script, we also run tests with a different type of storage device in another machine. These tests were run in a Vostro 5740 laptop running 64 bits Linux 
(Ubuntu 16.04), using version 4.4.0 of the Linux kernel. This laptop had an Intel Core i54210U processor, with a $1.70 \mathrm{GHz}$ clock, and $4 \mathrm{GiB}$ of RAM. In this machine we ran tests on a SD card attached to an integrated card reader, referenced in the text as SDCARD:

SDCARD: SandDisk Ultra SD card (32 GB).

In this device the tests were run in a dedicated $1.44 \mathrm{GiB}$ partition. Although this device is not representative of the type of devices usually employed to support distributed algorithms, we found that the data obtained helps to support some observations we made as more general and applicable to a wide range of devices.

Even though our test load uses synchronous writes to ensure data was stable in the drive before the application can continue, we do not bypass the file system and go direct to the device. Thus, the performance of the file system is also a significant factor in overall performance of the target applications. To assess how big this factor is, we selected a representative set of current file systems: XFS [Sweeney et al. 1996], ext4 [Mathur et al. 2007], BTRFS [Rodeh et al. 2013] and F2FS [Lee et al. 2015]. XFS and ext4 represent modern implementations of a "classic" file system, while BTRFS is a more recent design. F2FS is a log-structured file system tailored for flash-based devices.

\section{Results}

In this section we first present the throughput and latency data for each of the devices and file systems tested. Using this data we show how to choose a buffer size appropriate for a specific distributed algorithm. All charts in this section have logarithmic-scaled $x$ axis, because data on buffer size was measured by doubling the buffer size. This way we can cover a larger range of buffer sizes in less time, but this distorts the data. To compensate for this, the $y$ axis is also in logarithmic scale. In our discussion of the results, when comparing two file systems we use an independent t-test to test for statistic significance, with $p<0,001$ as threshold.

\subsection{Hard Disk}

The results for the HDD device are shown in Figure 1 (throughput) and Figure 2 (latency), comparing the XFS, ext4 and BTRFS file systems. The first observation we can make is that, as expected, throughput increases proportionally to buffer size while latency remains mostly constant for small buffer sizes. In this range, performance is dictated by synchronous write performance of the device and efficiency of the file system. As buffers increase, however, latencies start to raise as memory and disk throughput start having a larger impact on the performance. For larger buffers, throughput remains constant while latency increases proportionally to buffer size. This behavior is also expected, as a throughput-saturated disk will take more time to write a larger buffer.

The behavior considering very small and very large buffers is predictable, while the transition between the two is much less so. In Section 4.4 we will discuss this transition in more depth, but first we are going to make some observations about the performance of the file systems in the HD device. For small buffers, BTRFS has the worst latency, XFS has the best, at about half of the latency of BTRFS, and ext4 stays in the middle. This latency dominates the throughput and the relative performance of these file systems stays roughly the same for small buffers: XFS is the best, followed by ext4 and 


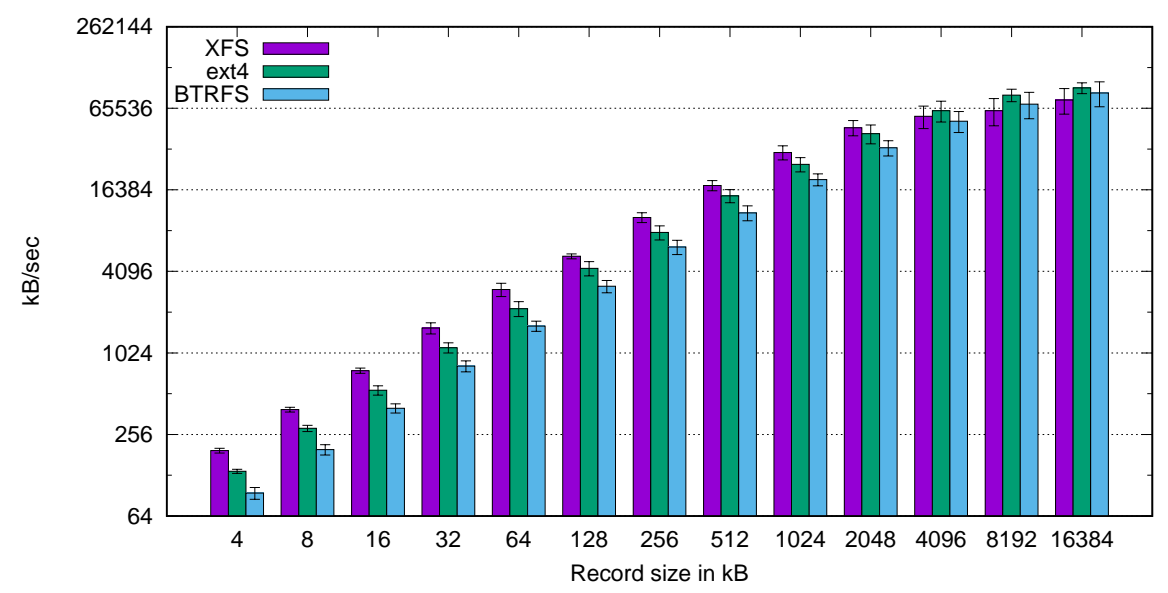

Figure 1. HDD Throughput

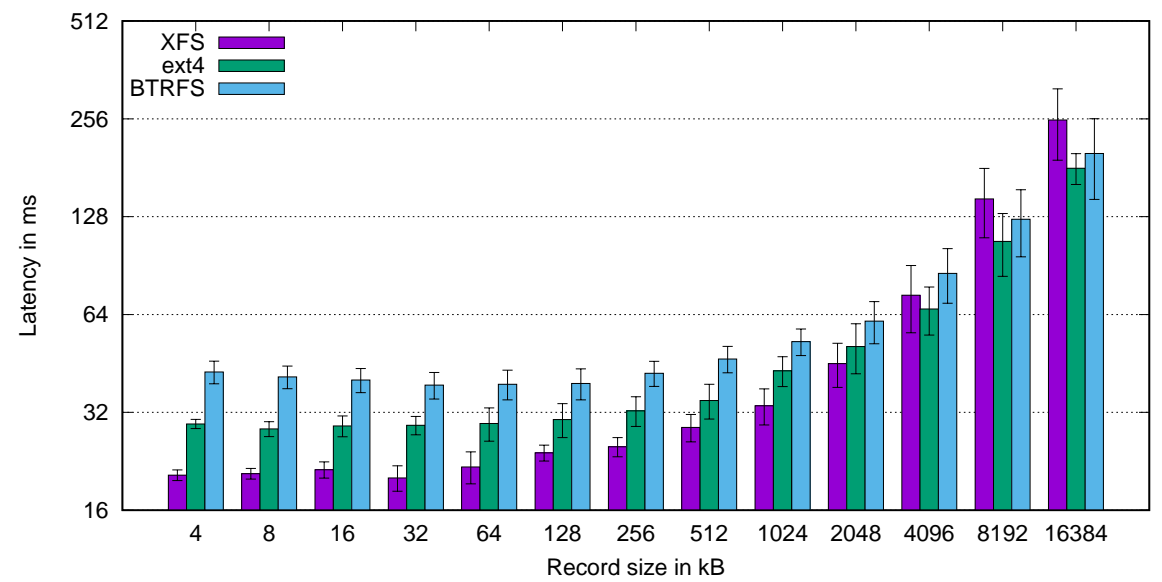

Figure 2. HDD Latency

BTRFS. We don't have data to support this, but we speculate this difference in performance is due to the management of metadata for each file system, with BTRFS showing the largest overhead.

For larger buffers we are approaching the maximum throughput of the underlying device. With a saturated disk file system differences start to disappear and optimizations to writing of bulk data and metadata start to make a difference. This appears to be specially true for BTRFS, which takes advantage of larger file system blocks [Rodeh et al. 2013]. There are still some noticeable differences in the averages, but the standard deviation increased as well. For instance, for the buffer size of $4096 \mathrm{kB}$ that marks a point where the average throughput of ext4 surpasses the throughput of XFS, this difference is not statistically significant $\left(t_{(30)}=2,02, p=0,049\right)$.

\subsection{SSD}

The results for the SSD device are shown in Figure 3 (throughput) and Figure 4 (latency), comparing the XFS, ext4, BTRFS and F2FS file systems. The data confirms that, despite their considerable difference in implementation, both HDD and SSD share a very similar performance profile. We can observe increasing throughput with constant latency 
for smaller buffers, and constant throughput with increasing latency for larger buffers. What takes HDD and SSD apart is the magnitude of the performance, with throughput and latency of SSD about $4 x$ better. Moreover, the SSD device reaches its maximum throughput with smaller buffers than the HDD. This means that this class of device has a real advantage for use with distributed algorithms because the maximum throughput can be achieved with lower latency, as we discuss in Section 4.4.

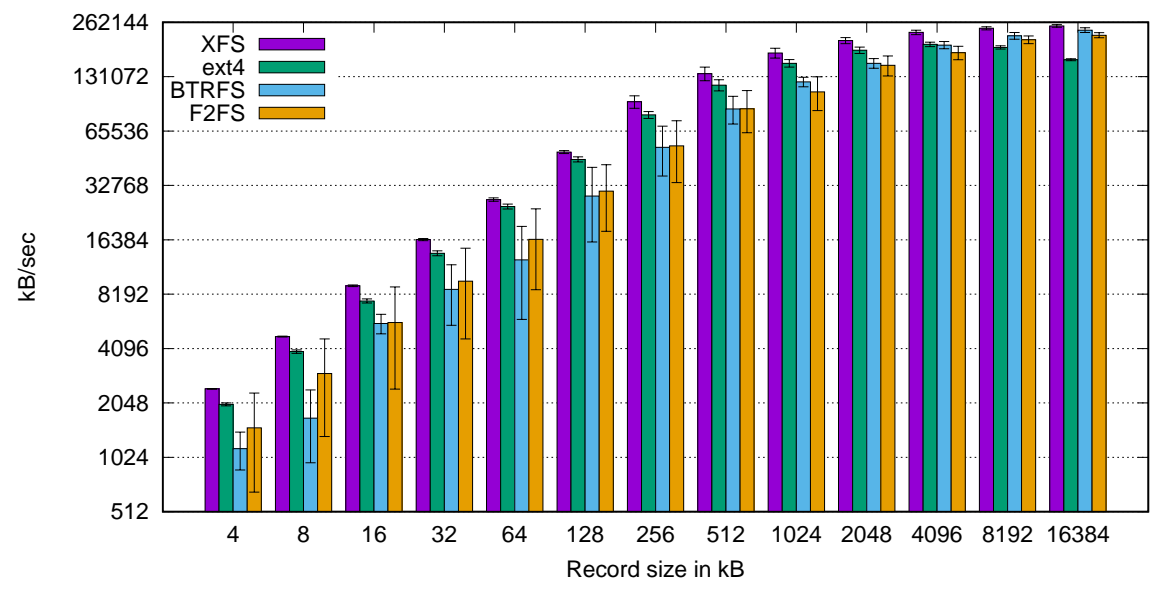

Figure 3. SSD Throughput

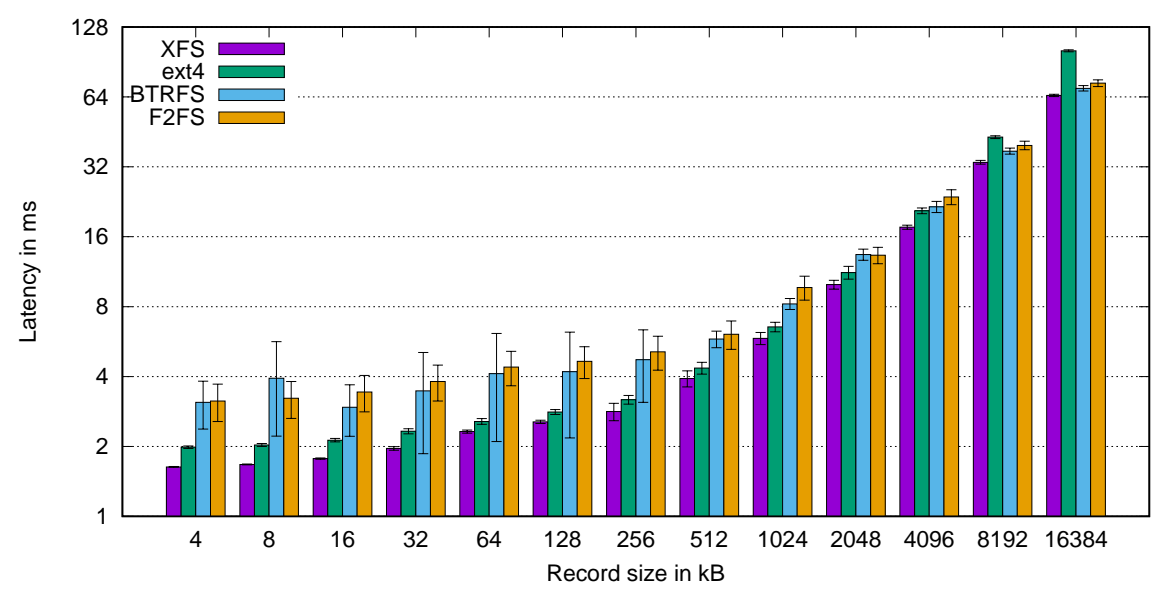

Figure 4. SSD Latency

Regarding the relative performance of the tested file systems, we observed in the SSD device a similar pattern found in the HDD device. For small buffer sizes, XFS and ext4 have a clear advantage, with XFS leading by a small margin. For example, with buffer size of $128 \mathrm{kB}$ XFS achieves $50127 \mathrm{kB} / \mathrm{s}$ while ext4 manages $45675 \mathrm{kB} / \mathrm{s}$, a statistically significant difference $\left(t_{(30)}=13,8, p<0,001\right)$. Both BTRFS and F2FS have weak performance with small buffers, probably due to metadata overhead. F2FS in particular buffers concurrent synchronous writes and is not particularly suited for the single-threaded workload we tested [Lee et al. 2015]. Moreover, BTRFS and F2FS show a very high standard deviation, probably indicating that metadata overhead is not constant and probably has some infrequent high-cost operations. 
As observed for the HDD, as we increase the size of the buffers for the SSD, file systems differences tend to get smaller. But, as the SSD device shows a smaller standard deviation, these differences are more consistent. For example, for buffer size of $4096 \mathrm{kB}$ the throughput of XFS is $230612 \mathrm{kB} / \mathrm{s}$ while the throughput of ext4 is $197912 \mathrm{kB} / \mathrm{s}$, a statistically significant difference $\left(t_{(30)}=20,8, p<0,001\right)$. One unexpected observation was that ext4 had a noticeable and consistent drop in throughput for the largest buffers. We don't have data to support it, but we speculate as the throughput increases ext4 uses more CPU and may start to be CPU bound.

\subsection{SDCARD}

The results for the SDCARD device are shown in Figure 5 (throughput) and Figure 6 (latency), comparing the XFS, ext4, BTRFS and F2FS file systems. The SDCARD device is a low capacity, removable device, with a basic FTL (flash translation layer [Chen et al. 2009, Min et al. 2012]) and consequently lower throughput and higher latency than the SSD device. The data confirms a low maximum throughput, but the device is able to reach this throughput with smaller buffers. As a consequence, at the maximum throughput the latency is surprisingly low.

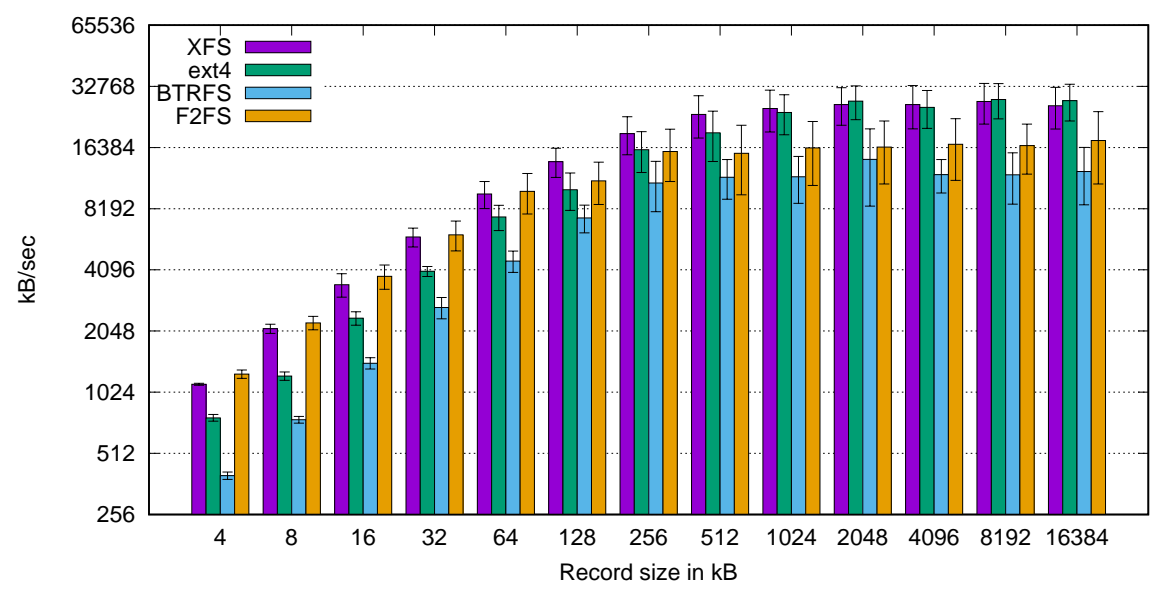

Figure 5. SDCARD Throughput

F2FS has specially low latencies up to $32 \mathrm{kB}$ buffers and consequently a higher throughput, beating XFS by a small margin. This difference is only statistically significant up to $8 \mathrm{kB}$ buffers $\left(t_{(30)}=3,9, p=0,0002\right)$. Nonetheless, this shows the F2FS file system is particularly optimized for this class of storage device.

For larger buffer sizes, F2FS disappointingly plateaus at about two thirds of the final throughput achieved by both XFS and ext4. For these larger buffer sizes the standard deviation increases considerably and performance differences between XFS and ext4 are not statistically significant. With a buffer size of $1 \mathrm{MB}$ XFS reaches $25469 \mathrm{kB} / \mathrm{s}$ while ext4 throughput is $24368 \mathrm{kB} / \mathrm{s}$, a not statistically significant difference $\left(t_{(30)}=0,75, p=\right.$ $0,454)$. XFS is however better than F2FS for larger buffers. For the same 1 MB buffer F2FS throughput is $16321 \mathrm{kB} / \mathrm{s}$, and XFS has a statistic significant advantage $\left(t_{(30)}=\right.$ $6,12, p<0,001)$. 


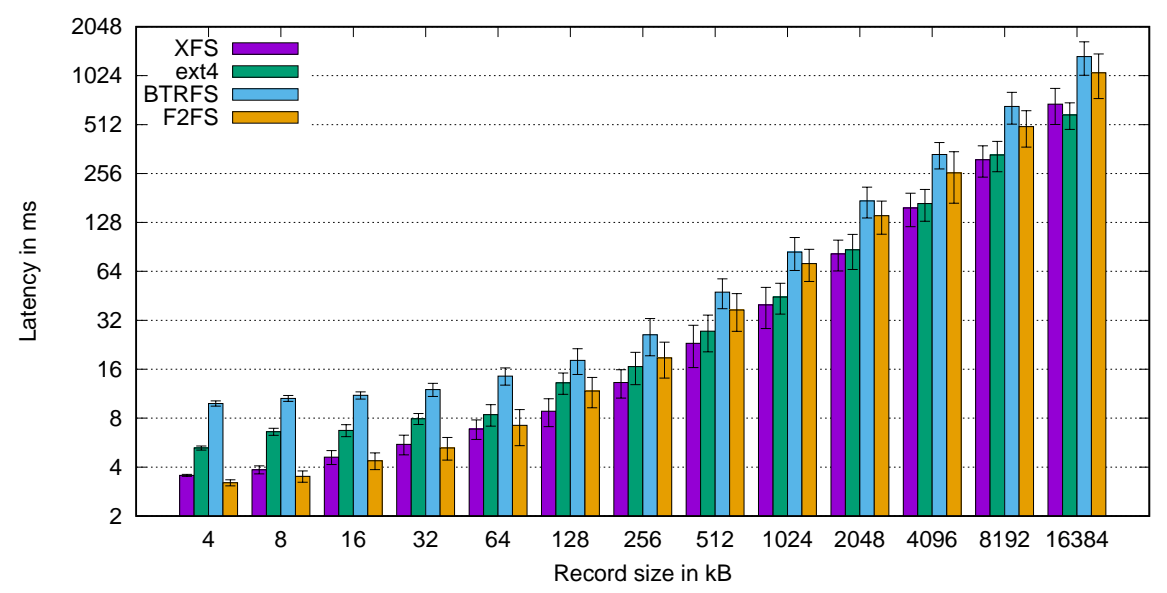

Figure 6. SDCARD Latency

\subsection{Balancing Throughput and Latency}

With the throughput and latency curves obtained with our benchmark a system designer can pick the required buffer size for implementation of a distributed algorithm. If the application needs to move data with a minimum throughput, the designer should choose the smallest buffer that achieves the desired throughput, if possible. If the application requires responses to be sent with a maximum latency, the designer should choose the largest buffer that doesn't violate this limit, if possible. However, matters aren't as clear if the application hasn't any hard limit on throughput and latency, but only a general desire to optimize the balance between the two.

Each metric we have measured has a transition: throughput increases until it reaches maximum device throughput and then stabilizes; latency is stable until transfer costs start to dominate, then it starts to increase. The two transition points do not coincide, they happen in different buffer sizes. For example, considering XFS on the HDD the throughput starts to level off at buffer sizes of $4096 \mathrm{kB}$ to $8192 \mathrm{kB}$, while latency starts picking up with buffer sizes of $512 \mathrm{kB}$ to $1024 \mathrm{kB}$.

To try and capture the tradeoff between throughput and latency in face of the fact that the tipping point of each metric is different we introduce a combined metric, the ratio of throughput by latency. This new metric gives a rough idea of the efficiency of a buffer size, indicating how many units of throughput one can gain for each unit of latency introduced. Figures 7, 8 and 9 show this new metric for the devices and file systems tested.

In a sense, the curve observed in these figures can be seen as a combination of the throughput and latency curves. The ascending slope represents the phase which increasing buffer size will increase throughput more than it increases latency. The plateau is the phase where latency and throughput increase in the same rate. The descending slope represents the phase where the increase in throughput in smaller than the increase in latency. The maximum of the curve represents the best throughput for each unit of latency. If one considers larger buffers, the latency is increased but the throughput won't improve. This is the point where throughput is maximized requiring the least of latency.

Using this metric as guide, we can observe that the SSD device is more capable 


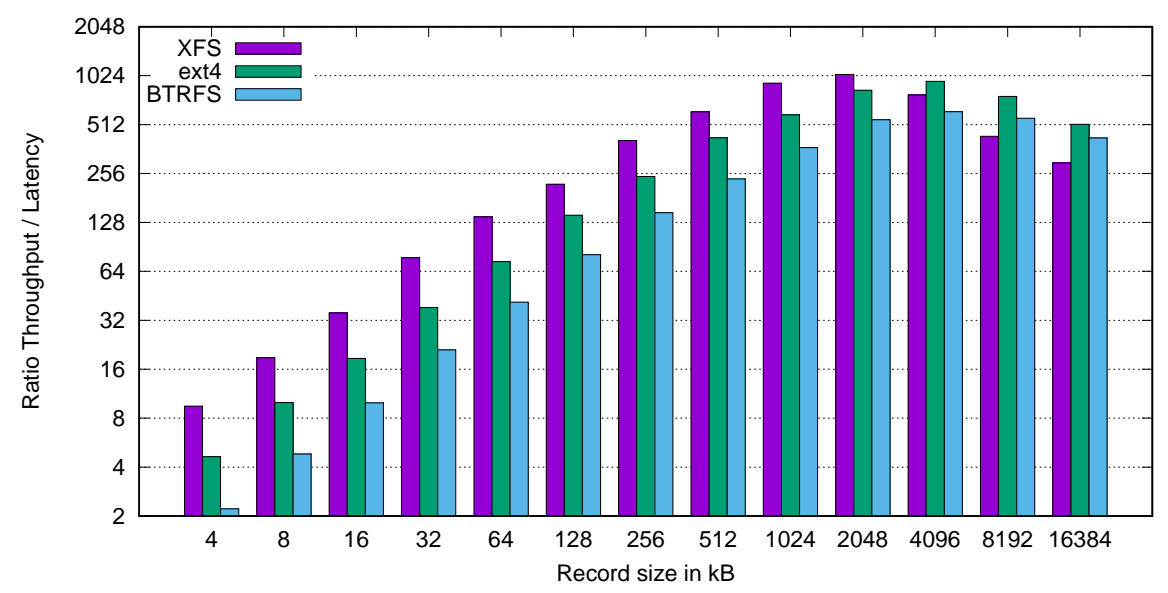

Figure 7. HD Ratio

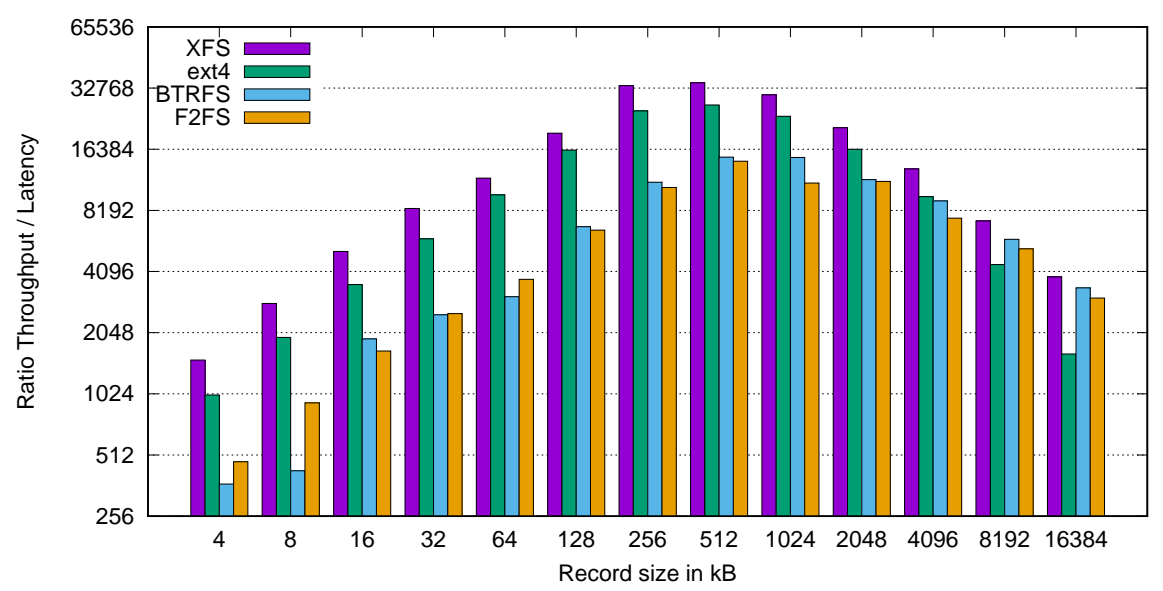

Figure 8. SSD Ratio

of handling the loads of distributed algorithms because it can saturate its write throughput with smaller buffers. The SSD has a superior absolute performance, but the ratio of throughput/latency is more relevant in this case. As an example of this, take the SDCARD device. As a low end device, without the sophisticated FTL found in the SSD, its performance parameters are arguably inferior to the HDD. Maximum throughput of the SDCARD is about half of the HDD, but latency is about half. As a consequence, the optimum throughput/latency ratio is about the same for both devices, but with the SDCARD showing latencies that are 10x smaller. Throughput is lower, however, but only about $4 \mathrm{x}$ lower. Thus, for an application in which this lower throughput is acceptable, surprisingly the SDCARD device would be an interesting choice.

\section{Conclusion}

Distributed algorithms in the fail-recovery failure model require efficient access to stable memory. This efficiency is measured by the trade-off between the latency of each write to stable memory and the total throughput of writes. A distributed system programmer wants to balance the two by correctly sizing the buffers sent to be written by the file system, ideally achieving a target throughput with the minimum latency possible. To 


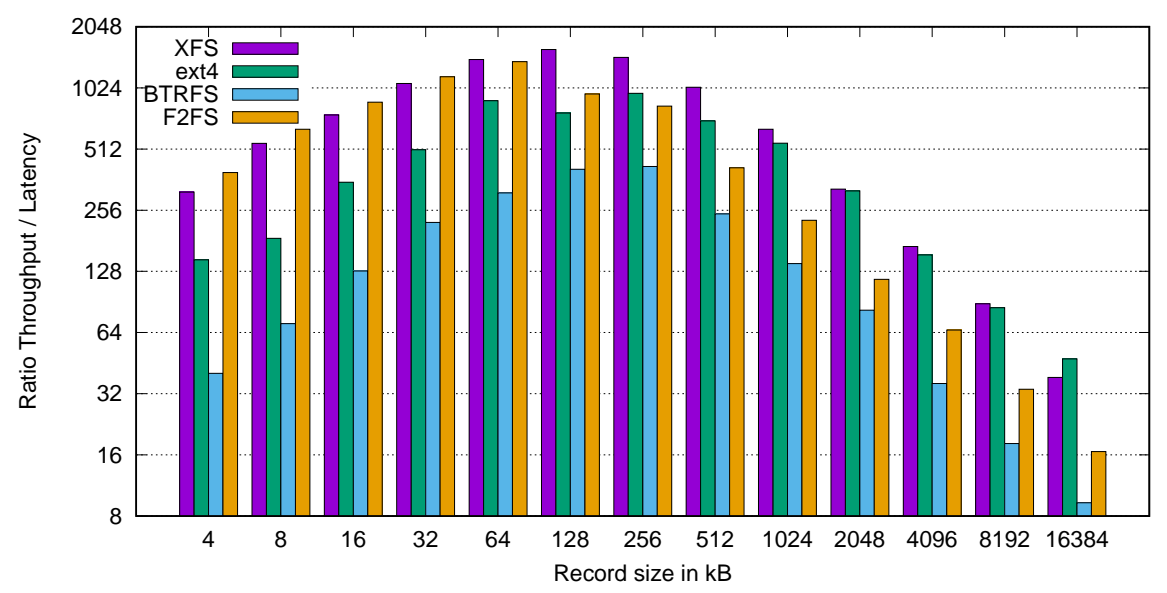

Figure 9. SDCARD Ratio

aid in this task, we have characterized the performance profile of typical stable memory devices, a spinning disk and a solid state drive, using a representative set of current file systems.

Our data show that the performance of the studied storage devices show three distinct phases as application buffer size grows. In the first phase throughput increases while latency stays approximately constant. In the second phase throughput and latency increase proportionally. In the third phase throughput reaches a maximum and latency starts increasing. In general terms, a designer should choose a buffer size in the first state with the minimum latency that respects the required throughput of the application.

With respect to the devices tested, the ones that saturate the throughput with smaller buffer sizes tend to offer the smaller latency. The SSD device was the best in this respect, with very good throughput and latency figures. Surprisingly, the SDCARD device showed a very interesting balance between throughput and latency, despite being a low performance device. With respect to file systems, the ones that handle large buffers efficiently were the best performers. In particular, XFS showed a very consistent performance.

\section{References}

Cachin, C., Guerraoui, R., and Rodrigues, L. (2011). Introduction to reliable and secure distributed programming. Springer.

Chandra, T. D., Griesemer, R., and Redstone, J. (2007). Paxos made live: an engineering perspective. In PODC '07: Proceedings of the twenty-sixth annual ACM symposium on Principles of distributed computing, pages 398-407, New York, NY, USA. ACM Press.

Chen, F., Koufaty, D. A., and Zhang, X. (2009). Understanding intrinsic characteristics and system implications of flash memory based solid state drives. SIGMETRICS Perform. Eval. Rev., 37(1):181-192.

Hunt, P., Konar, M., Junqueira, F. P., and Reed, B. (2010). ZooKeeper: Wait-free coordination for internet-scale systems. In Proceedings of the 2010 USENIX Conference on 
USENIX Annual Technical Conference, USENIXATC'10, pages 11-11, Berkeley, CA, USA. USENIX Association.

Jannen, W., Yuan, J., Zhan, Y., Akshintala, A., Esmet, J., Jiao, Y., Mittal, A., Pandey, P., Reddy, P., Walsh, L., Bender, M., Farach-Colton, M., Johnson, R., Kuszmaul, B. C., and Porter, D. E. (2015). BetrFS: A right-optimized write-optimized file system. In Proceedings of the 13th USENIX Conference on File and Storage Technologies, FAST'15, pages 301-315, Berkeley, CA, USA. USENIX Association.

Lamport, L. (1998). The part-time parliament. ACM Trans. Comput. Syst., 16(2):133169.

Lamport, L. (2006). Fast Paxos. Distrib. Comput., 19(2):79-103.

Lee, C., Sim, D., Hwang, J.-Y., and Cho, S. (2015). F2FS: A new file system for flash storage. In Proceedings of the 13th USENIX Conference on File and Storage Technologies, FAST'15, pages 273-286, Berkeley, CA, USA. USENIX Association.

Mathur, A., Cao, M., Bhattacharya, S., Dilger, A., Tomas, A., and Vivier, L. (2007). The new ext4 filesystem: current status and future plans. In Proceedings of the Linux symposium, volume 2, pages 21-33.

Min, C., Kim, K., Cho, H., Lee, S.-W., and Eom, Y. I. (2012). SFS: Random write considered harmful in solid state drives. In Proceedings of the 10th USENIX Conference on File and Storage Technologies, FAST'12, pages 12-12, Berkeley, CA, USA. USENIX Association.

Patterson, D. A. (2004). Latency lags bandwith. Commun. ACM, 47(10):71-75.

Rodeh, O., Bacik, J., and Mason, C. (2013). BTRFS: The linux B-tree filesystem. Trans. Storage, 9(3):9:1-9:32.

Rosenblum, M. and Ousterhout, J. K. (1992). The design and implementation of a logstructured file system. ACM Trans. Comput. Syst., 10(1):26-52.

Ruemmler, C. and Wilkes, J. (1994). An introduction to disk drive modeling. Computer, 27(3):17-28.

Schneider, F. B. (1990). Implementing fault-tolerant services using the state machine approach: a tutorial. ACM Comput. Surv., 22(4):299-319.

Sweeney, A., Doucette, D., Hu, W., Anderson, C., Nishimoto, M., and Peck, G. (1996). Scalability in the XFS file system. In Proceedings of the 1996 Annual Conference on USENIX Annual Technical Conference, ATEC '96, pages 1-1, Berkeley, CA, USA. USENIX Association.

Vieira, G. M. D. and Buzato, L. E. (2010). Implementation of an object-oriented specification for active replication using consensus. Technical Report IC-10-26, Institute of Computing, University of Campinas.

Yeon, J., Jeong, M., Lee, S., and Lee, E. (2018). RFLUSH: Rethink the flush. In Proceedings of the 16th USENIX Conference on File and Storage Technologies, FAST'18, pages 201-209, Berkeley, CA, USA. USENIX Association. 